\title{
Museo de Arte Moderno de Trujillo
}

Rodolfo Cortegana

Es muy dificil aproximarse criticamente a un museo cuando la ausencia de iniciativas para crearlos se encuentran con la filantropía de un proyecto personal. El Museo de Arte Moderno de La Ciudad de Trujillo nos obliga a plantearnos la interrogante de si es suficiente la buena voluntad para hacer uno. La respuesta en este caso es complicada y supone hacer algunas consideraciones sobre la arquitectura y los lugares para la construcción de museos que serán el motivo de esta nota.

En la década de los años 80 el museo se jerarquizó como el edificio emblemático en donde se resguardaba la memoria de la sociedad. En esos años, justamente, se realizaron tres bienales importantes (1983-85-87) en la Ciudad de Trujillo, donde Gerardo Chávez tuvo una participación relevante, junto con un grupo de artistas locales, actividad que sin duda sería un importante precedente del monumental proyecto desarrollado años después por el connotado artista.

La propuesta de la Bienal se caracterizó por tomar las casonas del centro de Trujillo y otros locales, adaptarlos y ocuparlos como espacios de exhibición. El centro de la ciudad fue inundado de arte y los pobladores vivieron de manera cotidiana esta fiesta. Esto generó que una serie de locales, ubicados muy cerca de la Plaza de Armas, se transformaran en Galerías de Arte o espacios culturales, cuya efervescencia declinó a finales de los 80 y terminó casi por desaparecer en los 90 e inicios del 2000.

En otros contextos la década del 80 marcó el inicio de la creación de grandes edificaciones que por si mismas regeneraban la ciudad y cambiaban por completo la situación de degradación de algunos barrios, tal como ocurrió en París con la construcción del Centro Cultural George Pompidou y la pirámide del Museo de Louvre; con el Museo de Arte Moderno de Barcelona construido en los 90 y en este nuevo siglo con el Museo Guggenheim de la ciudad de Bilbao. Eran los sueños de algunas ciudades que apostaron a través de estas estructuras, algunas monumentales, a consolidar una presencia artística para ellas. Con los años esta idea ha ido dando paso a otras propuestas no menos interesantes y más sostenibles como el MUSAC (Museo de Arte Contemporáneo de Castilla), que integra, a partir de edificaciones de escala pequeña, a un espacio público generando una continuidad en el perfil de la ciudad. 


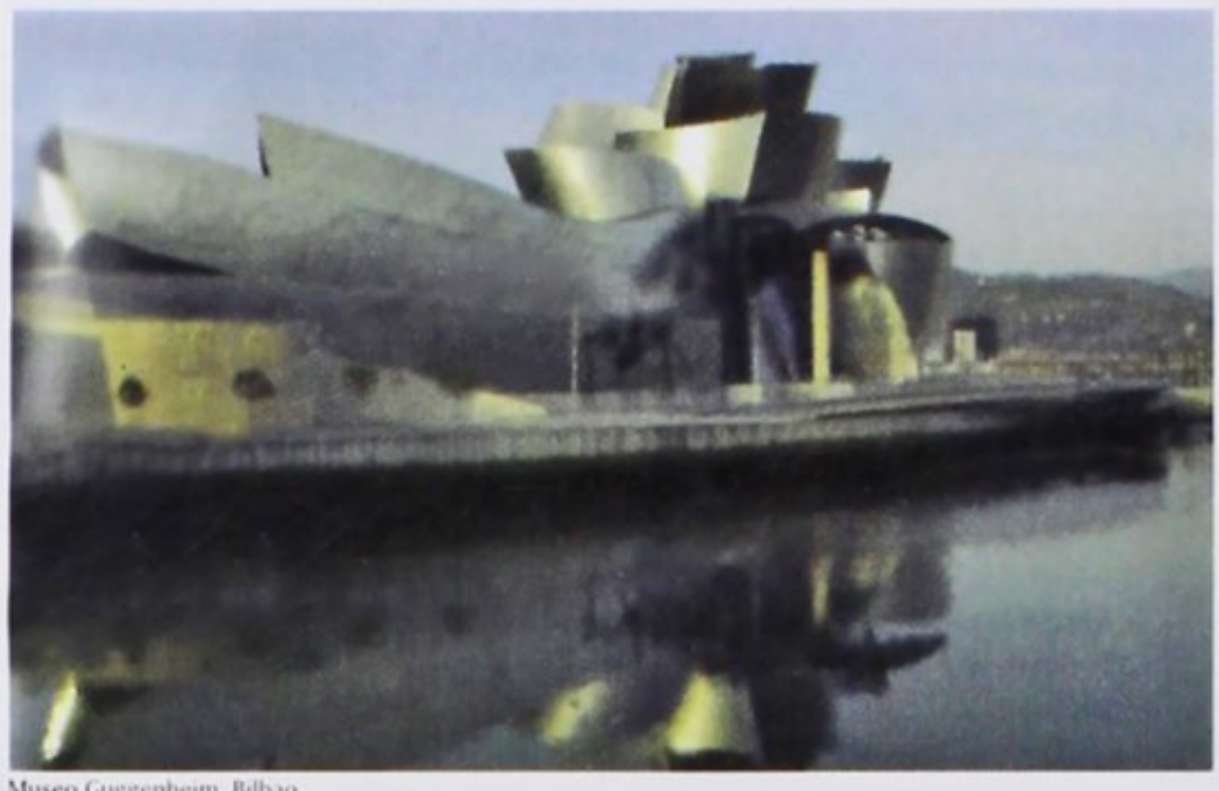

El museo entonces si puede ser un referente para el desarrollo de algunos barrios de la ciudad, pero siempre a partir de un sistema planificado $y$ del aprovechamiento de referentes patrimoniales que le permitan recobrar y potenciar ciertas identidades. El tema ya no pasa por la construcción de grandes edificaciones en la ciudad que luego terminan consumiendo enormes cantidades de energia y el mantenimiento se hace imposible a corto plazo; la reactivación de edificaciones antiguas y sus reciclajes o ampliaciones de museos existentes, sin dejar de la lado la construcción de edificaciones pertinentes, marcan la pauta para el vínculo con el espacio público y la regeneración de las ciudades.

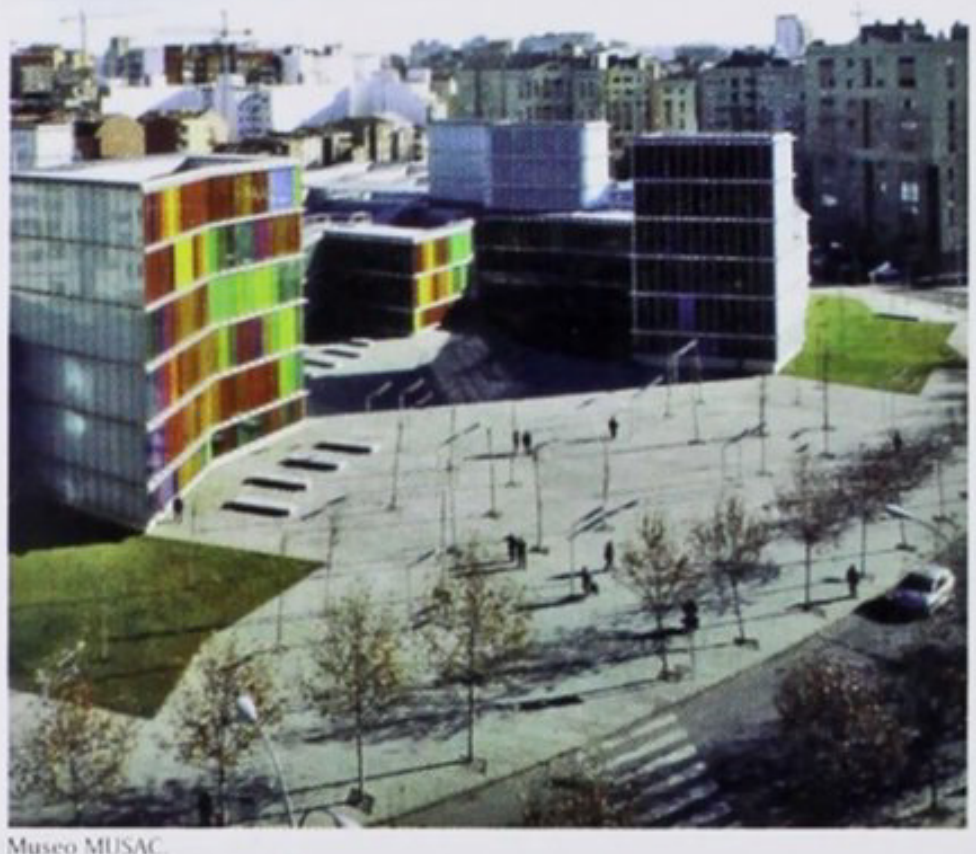

Hace muy pocos años hemos presenciado la construcción de algunos nuevos museos en el norte del pais que fueron planteados a partir de grandes monumentos que no han logrado activar la regeneración de sus alrededores; me estoy refiriendo al museo Tumbas Reales de Sipán en Lambayeque y al Museo Sicán en Ferreñafe, que se crearon como hitos urbanos y que no han sido capaces de relacionar el entorno urbano con la cultura alli expuesta.

La Ciudad de Trujillo, como lo mencionamos anteriormente. construyó una huella y una memoria con las bienales realizadas en la década de los 80 , demostrando que en la trama urbana de la ciudad y de sus inmuebles se puede reactivar una dinámica relacionada con el arte a partir de la intervención, ampliación o construcción de algunas edificaciones del centro histórico.

La presencia de una Escuela de Bellas Artes puede ser un detonante para la aparición de espacios culturales, la ciudad puede entonces construir un eje hacia el centro desde la Escuela aprovechando esta situación. Creo que todos los esfuerzos deben de estar vincu- 
lados a la población, su ciudad y espacios públicos. El Museo como ente privado no se puede librar de esta condicionante.

Nadie puede negar el gran esfuerzo realizado, casi de manera personal por el artista Gerardo Chávez, para construir una entidad como el Museo de Arte Moderno de Trujillo; sin embargo, quisiéramos anotar algunas consideraciones sobre el

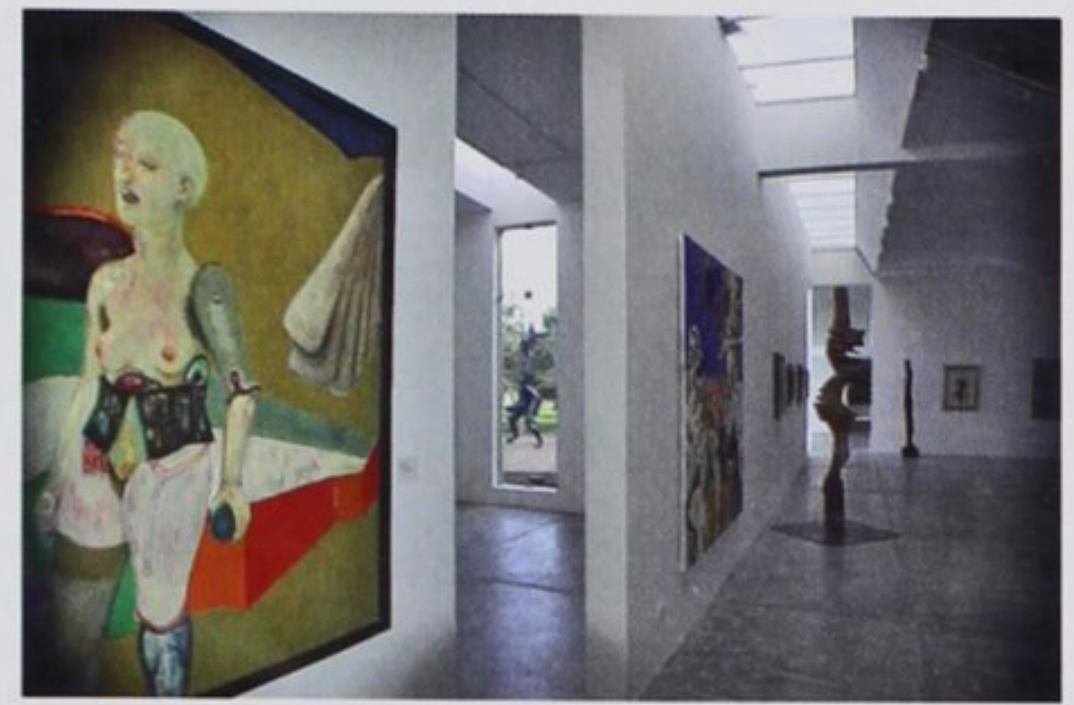

Interiores del Museo de Arte Moderno de Trujillo. uso y diseño del espacio arquitectónico y de su entorno, que confrontan al proyecto con una nueva manera de ver el museo y su estrecha relación con la ciudad y sus pobladores.

A diferencia de lo mencionado líneas arriba con respecto a otras construcciones del país, el Museo de Arte Moderno en sus casi $1,500.00 \mathrm{~m} 2$, plantea una espacialidad que llamaremos vivencial, desligada de cualquier pretensión formal exterior e interior, ya que la propuesta apunta a un diálogo fluido y relacional entre la obra de arte (colección) y el espacio que la contiene. Desde el inicio del recorrido un espacio acentuado por una bóveda de cañón muy delgada nos recibe jerarquizando de esa manera una pieza notable del escultor suizo Alberto Giacometti, la cual centra el eje visual definido por el espacio, cuya importancia revela el afecto personal que tiene para el artista esta singular obra. Las preocupaciones se destacan de inmediato: Gerardo Chávez y el Arq. Guillermo Morales han encontrado. una clara manera de exponer una colección personal que se lee de manera adecuada; es indiscutible la pertinencia de cómo un artista se involucra en una edificación arquitectónica, llevando de la mano al espacio que tiene que servir de contenedor a la obra expuesta. Cuando hablamos del espacio vivencial, nos referimos a que el espacio arquitectónico es producto de una relación vivida, una experiencia que se lleva a cabo desde el interior y que se proyecta en términos de vínculos con el objeto allí expuesto; el espectador reconoce la obra y al mismo tiempo se reconoce en un espacio que la ilumina y la recrea.

El espacio arquitectónico es entonces una celebración para lo expuesto y el tema de la luz, fundamental en un museo, se controla desde el interior de las salas para no perturbar los cuadros y al mismo tiempo hacer referencia a un evento exterior. Se hace importante la protección contra rayos ultravioletas a través del uso de

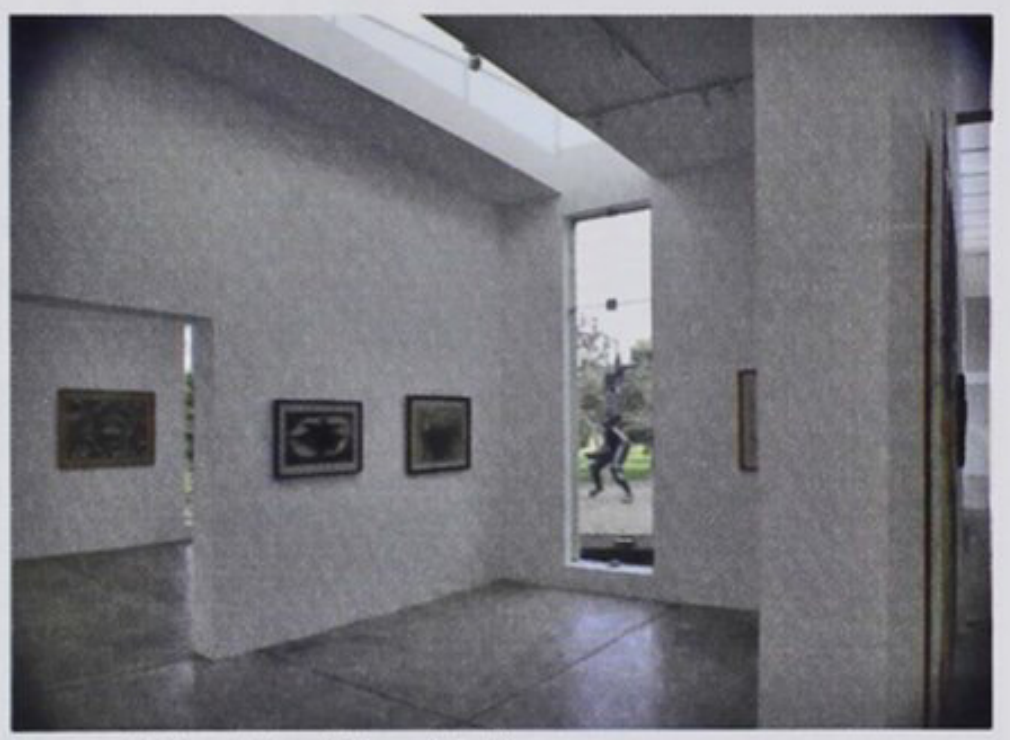




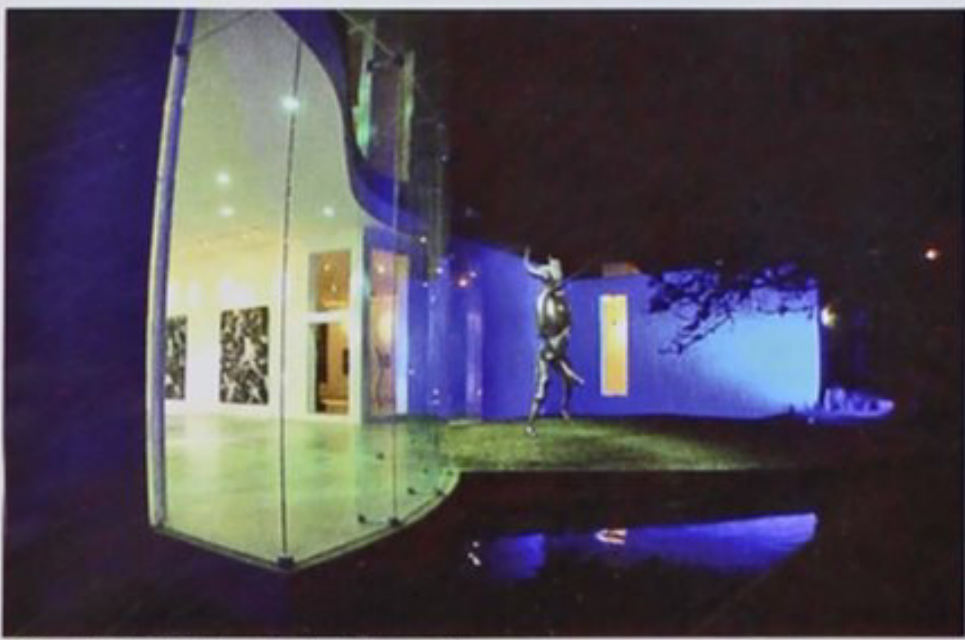

Museo de Arte Modemo de Trujillo de noche. policarbonato de $10 \mathrm{~mm}$ con protección UV para todos los ingresos de luz cenital que se tienen en el Museo.

La posibilidad de tener un espacio exterior con algunas obras vinculadas visualmente al interior, a través de grandes vanos, permite la fluidez en la percepción del espacio. Nota aparte merece la sala donde se expone el gran cuadro de Gerardo Chávez "La Procesión de la papa", de 12 metros de largo, cuyo emplazamiento ha generado el espacio y activado la arquitectura, logrando que la escala utilizada sea la adecuada y nos permita observar la pieza en toda su magnitud y potencia.

Otro tema importante y algo contradictorio, me parece la manera como la materialidad no refleja su verdadera expresión; se ha intentado imitar el adobe o un material parecido aplicando una textura en su exterior que simula dicho material, entonces nos debemos preguntar ¿es contemporánea toda la arquitectura actual? Me parece que este Museo refleja el poco conocimiento que se tiene de las construcciones del pasado y sus materiales. Un referente interesante para poder hacer arquitectura contemporánea, la materialidad de la arquitectura, nos abre distintos referentes y no solo con materiales, digamos nobles, sino más bien con indagaciones reales en la utilización de materiales como el adobe, si esa era la intención. Justamente la arquitectura y el arte moderno se referian claramente al "espíritu de la época", como un detonante para su producción; de aquí lo débil de la materialidad de la edificación.

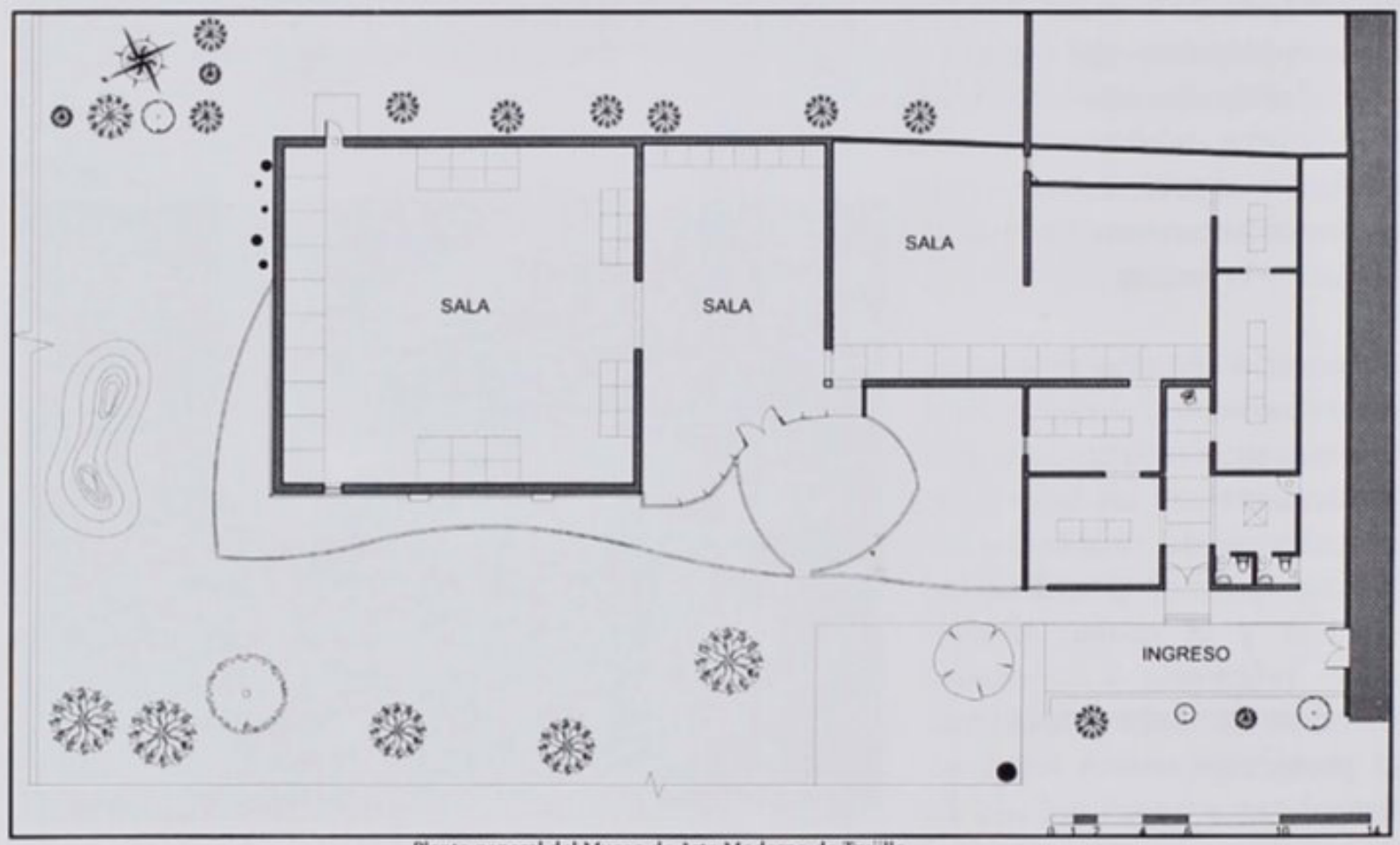

Planta general del Museo de Arte Moderno de Trujillo. 
Frente a esta situación con respecto al uso del material nos preguntarnos por otra variable de la arquitectura que tiene que ver con su emplazamiento: ¿Cuán importante es la ubicación de un museo en una ciudad? Sabemos que las gestiones realizadas por Chávez para poder realizar este proyecto en el centro histórico de la ciudad de Trujillo nunca llegaron a buen término y es por eso que se planteó el jardín de su casa,

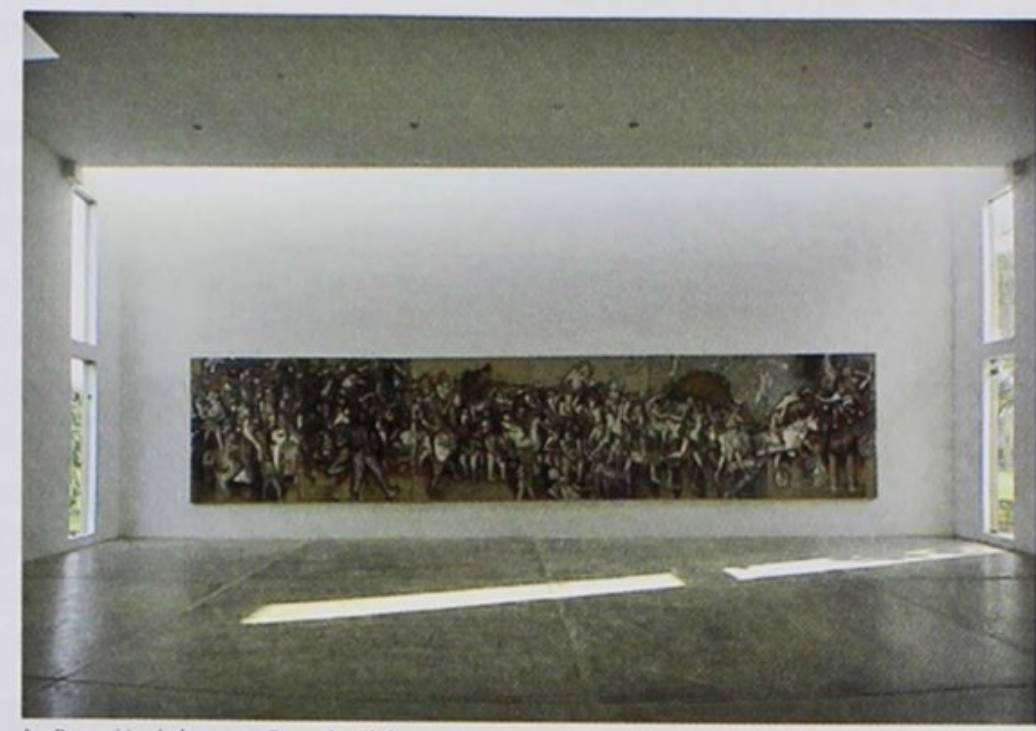

La Procesión de la papa, Gerardo Chávez.

a las afueras de la ciudad. De esta manera me parece que se perdió la posibilidad de que el museo sirviese para la reactivación de una zona degradada de la ciudad en el mismo centro, no sólo por su escala, sino porque estas intenciones siempre tienen que ser parte de una planificación que pueda. acompañar tamaño esfuerzo. ¿Habría podido intentar y conformar una iniciativa en el centro de la ciudad? Creo que los esfuerzos para que el museo pueda reactivar, en el centro histórico de la ciudad de Trujillo, un circuito en torno al arte eran posibles. El museo se hubiera convertido así en un detonante para la ciudad, reactivando el espacio público y generando un acercamiento real de la población con el arte de manera cotidiana.

El desplazamiento del objeto/obra de arte sacralizado ante el visitante/sujeto, nos puede abrir posibilidades en torno a cómo el museo se puede convertir en un espacio verdaderamente público, un espacio de contacto en donde las barreras para acercarse e ingresar a una colección no sean tantas. Es en este aspecto en donde encontramos el punto más débil de este esfuerzo, ya que quizás la enajenación del objeto moderno ensimisma la mirada del museo, en este caso privado, que puede ser construido en el jardín de una casa y no abrirse a la ciudad. Esta situación hace más dificil la posibilidad de que el público pueda encontrarse de manera natural con el espacio del museo; por eso es importante entonces la ubicación y, respondiéndome a la pregunta sobre ésta, es fundamental la elección del lugar para un edificio que, por su carácter altamente valorado por la sociedad, también corre el riesgo de estar altamente alejado de la misma. Creo que debemos convertir al museo en un espacio para la convivencia sin barreras que permita a la población acercarse a la obra de uno de los artistas más importantes de la escena cultural del país en la segunda mitad del siglo XX.

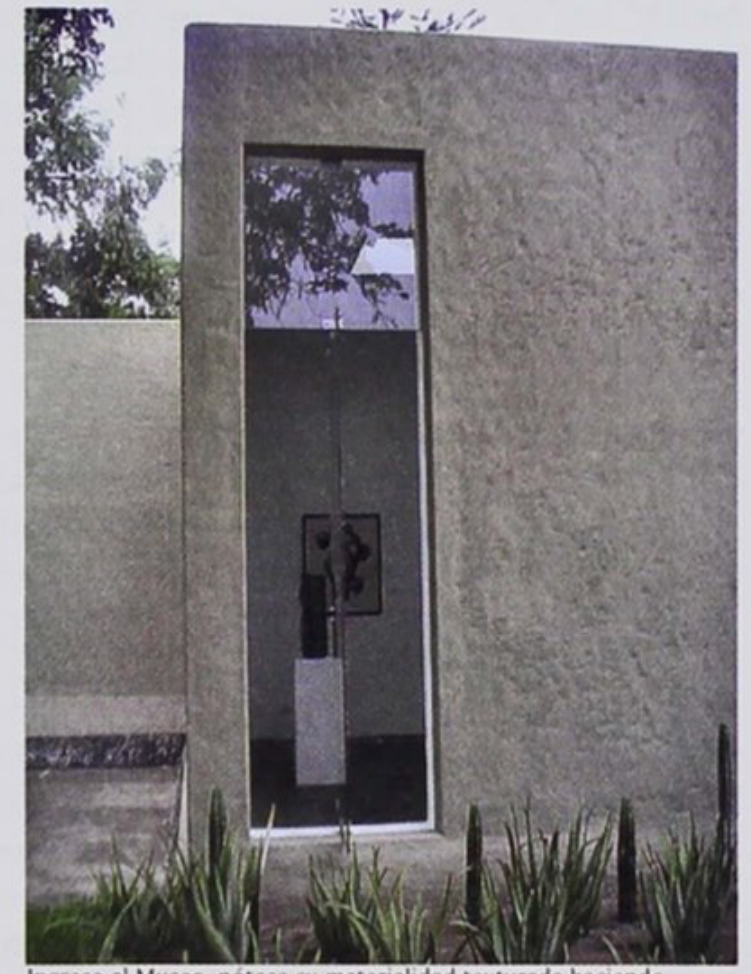

ngreso al Museo, notese su materialidad texturada haciendo alusión al adobe. 


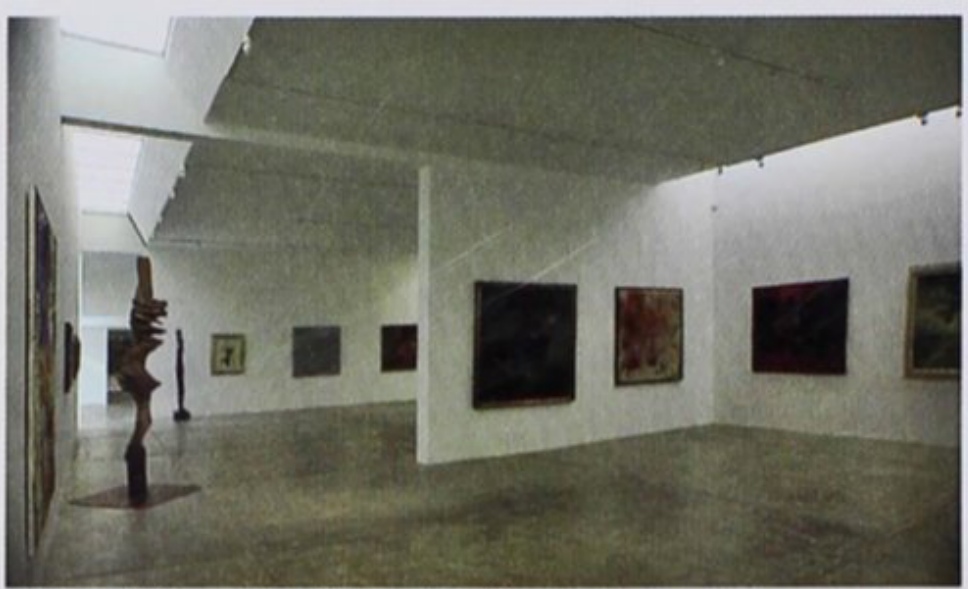

Interiores del Museo de Arte Moderno de Trujillo.

seriamente el tema. Se escuchan voces sobre la creación de nuevos museos en todo el país; es importante que las autoridades tengan a bien saber que el museo tiene su especificidad en el desarrollo del proyecto cultural, que no es una edificación cualquiera a la que se puede llenar de objetos para el día de la inauguración, sino que es el producto de un trabajo interdisciplinario en el cual se debe involucrar a toda la población en su realización.
Como anotaciones finales, ahora que es evidente cómo en el Perú se puede encontrar en sus autoridades regionales y municipales una vía para desarrollar proyectos culturales o museos, prevenirlos para que no vayan a jerarquizar necesidades políticas y anteponer el apoyo que puedan dar a iniciativas como el MAMT, por otras que desarrollan proyectos insostenibles, en donde no hay equipos que puedan trabajar

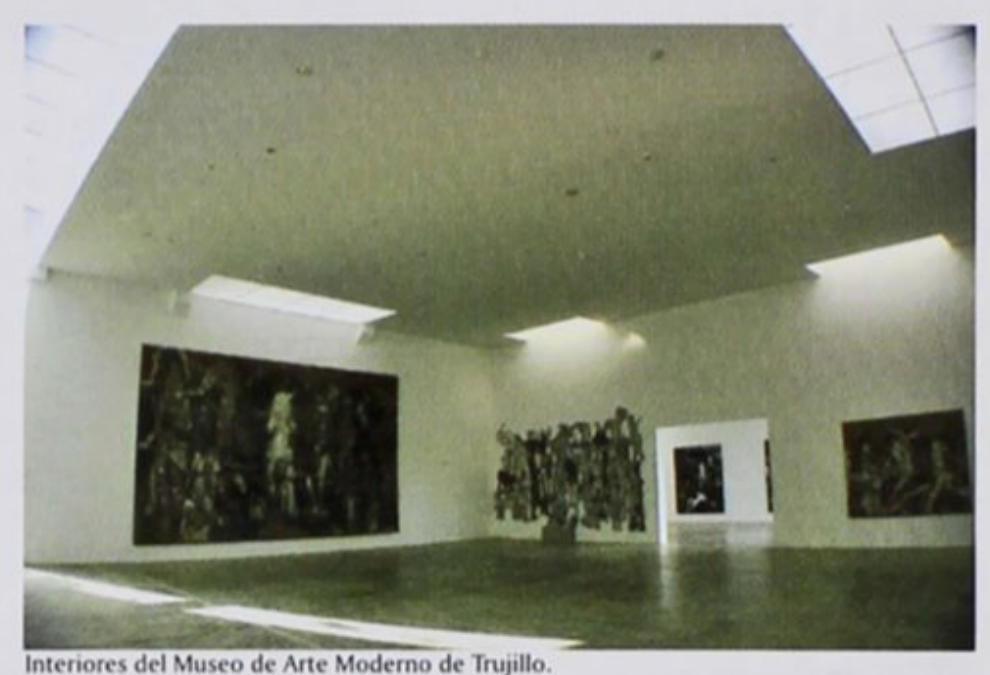

Interiores del Museo de Arte Moderno de Trujillo. 